\title{
Hadron structure beyond collinear level and precision DIS measurements
}

\author{
F Hautmann ${ }^{1,2,3}$ and $\mathrm{H}$ Jung $^{4,5}$ \\ ${ }^{1}$ Rutherford Appleton Laboratory \\ ${ }^{2}$ University of Oxford \\ ${ }^{3}$ University of Southampton \\ ${ }^{4}$ Deutsches Elektronen Synchrotron \\ ${ }^{5}$ University of Antwerp
}

\begin{abstract}
General formulations of QCD factorization for hadronic collisions extend the notion of ordinary parton distributions to transverse-momentum dependent (TMD) parton density and parton decay functions. We discuss the use of the recent high-precision deep-inelastic scattering (DIS) measurements for determination of TMD distributions. These are relevant for both low- $p_{T}$ and high- $p_{T}$ physics in hadron collisions. We comment on applications to multi-jet final states associated with electroweak gauge boson production at the LHC.
\end{abstract}

\section{Introduction}

Transverse momentum dependent (TMD) parton distributions extend the concept of ordinary (integrated) parton distribution functions (pdfs) to include transverse momentum and polarization degrees of freedom. They encode nonperturbative information on hadron structure which is essential in the context of QCD factorization theorems for multi-scale, non-inclusive collider observables. Classic examples are found in processes such as Drell-Yan (DY) production when the vector boson invariant mass is large compared to its transverse momentum, and deeply inelastic scattering (DIS) when the photon-hadron centerof-mass energy is large compared to the photon virtuality. In each of these cases, TMD pdfs obey evolution equations which generalize renormalization group evolution to the appropriate multi-scale regime. In each case such generalized evolution equations, once combined with factorization of the physical cross section in terms of TMD pdfs, allow one to resum logarithmically enhanced contributions to the perturbation series expansions for the physical observables to all orders in the QCD coupling.

A general program for TMD pdfs phenomenology was proposed in [1]. Recently, the TMDlib library has been started [2] to provide a platform for the collection and comparison of phenomenological studies of TMD pdfs. This aims to incorporate as broad as possible a set of processes and collect results from different approaches to TMD evolution and fitting. This includes e.g. partonmodel fits [3, 4] of low-energy data [5, 6]; CSS [7, 8] fits [9-11] of DY data [12, 13]; TMD fits [14-16] of highenergy DIS data [17-19].

This article focuses on the use of the precision DIS data from the combined HERA measurements [18, 19] to make a determination of the TMD gluon density [15] in- cluding, for the first time, experimental and theoretical uncertainties. The basis of this determination is the factorization of TMD pdfs in DIS at high energy. We briefly recall the main elements of this in Sec. 2. The results of the fits to precision measurements are summarized in Sec. 3. We discuss implications and give final comments in Sec. 4.

\section{DIS at high energy and factorization of TMD pdfs}

Consider deeply inelastic lepton-hadron scattering in the high-energy region $s \equiv 2 q \cdot p \gg Q^{2}$, where $p$ is the hadron four-momentum, $q$ is the four-momentum transferred by the lepton, and $Q^{2}=-q^{2}$. In this region the perturbation series expansions for the DIS structure functions $F_{j}(j=2, L)$ are affected by potentially large logarithms $\left(\alpha_{s} \ln s / Q^{2}\right)^{n}$ to all orders in the QCD coupling $\alpha_{s}$. TMD high-energy factorization for the structure functions [20] is pictured in Fig. 1. It is given in terms of two-gluon irreducible (2GI), perturbatively-calculable kernels and gluon (off-shell) Green's functions. The TMD gluon density is defined from the latter by using the high-energy projector $P_{H}[20,21]$ over spin and momentum.

The TMD factorization (Fig. 1) allows one to identify and sum the logarithmically enhanced corrections to coefficient functions and anomalous dimensions for $x=$ $Q^{2} /(2 p \cdot q) \ll 1$, fully taking into account the factorization scheme and factorization scale dependence. This has been applied to leading and next-to-leading logarithmic accuracy $[20,22,23]$.

The perturbation expansions in the flavor-singlet sector are single-logarithmic at high energy. For example, the gluonic hard-scattering coefficient function $C_{2}^{g}\left(N, \alpha_{s}, Q^{2} / \mu^{2}\right)$ for the DIS structure function $F_{2}$, as a 

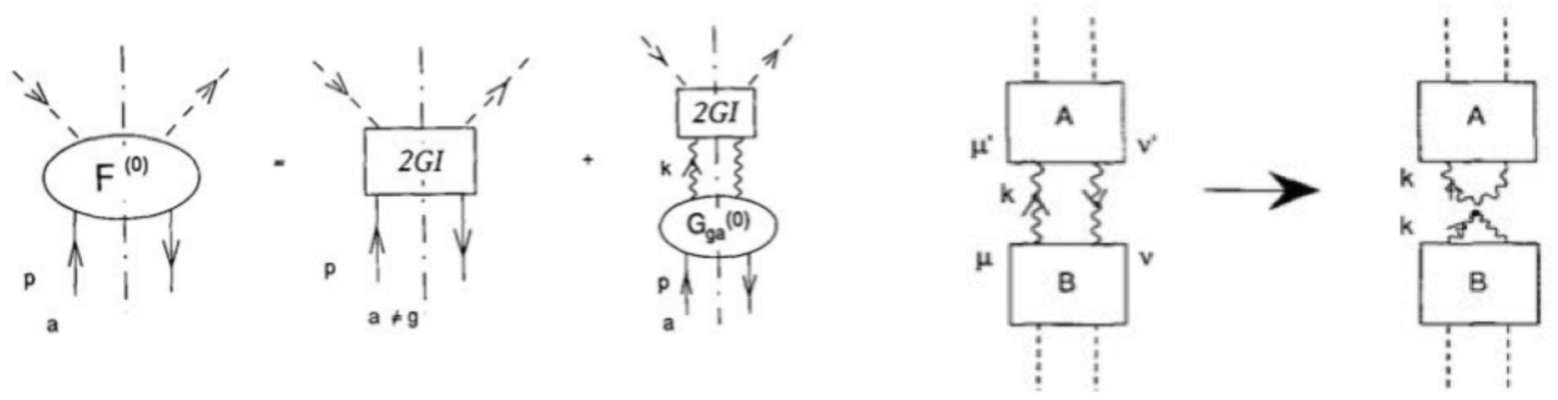

Figure 1. (left) Transverse momentum dependent high-energy factorization for DIS structure functions; (right) decoupling of 2 GI kernels via the high-energy projector.

function of the moment $N$ Mellin-conjugated to $x$, has the $\alpha_{s}$ expansion at scale $\mu^{2}=Q^{2}[20]$

$$
\begin{aligned}
& C_{2, N}^{g}\left(\alpha_{s}, Q^{2} / \mu^{2}=1\right) \\
& =\frac{\alpha_{s}}{2 \pi} T_{R} N_{f} \frac{2}{3}\left\{1+1.49 \frac{\bar{\alpha}_{s}}{N}+9.71\left(\frac{\bar{\alpha}_{s}}{N}\right)^{2}\right. \\
& \left.+16.43\left(\frac{\bar{\alpha}_{s}}{N}\right)^{3}+O\left(\frac{\alpha_{s}}{N}\right)^{4}\right\},
\end{aligned}
$$

where $\bar{\alpha}_{s}=\alpha_{s} C_{A} / \pi, C_{A}=3, T_{R}=1 / 2$, and the coefficients are given in the $\overline{\mathrm{MS}}$ minimal subtraction scheme. The $N \rightarrow 0$ poles $\alpha_{s}\left(\alpha_{s} / N\right)^{k}, k \geq 1$, correspond in $x$-space to next-to-leading-logarithmic (NLL) higher-loop corrections $\alpha_{s}^{2}\left(\alpha_{s} \ln x\right)^{k-1}$. With respect to the first two terms (one-loop [24] and two-loop [25], corresponding to leading order (LO) and next-to-leading order (NLO)), the next two terms (i.e., three-loop ${ }^{1}$ and four-loop) are logarithmically enhanced. Moreover, their numerical coefficients are significantly larger than the LO and NLO ones. The physical origin of the logarithmically enhanced higherloop terms lies with contributions to QCD multi-parton matrix elements from regions which are not ordered in the initial-state transverse momenta.

Given these results, there is little theoretical justification for treating the region $x \ll 1$ by truncating the perturbative expansion to fixed NLO (or NNLO) level. In this region fixed-order perturbative approaches, however successful phenomenologically, are theoretically disfavored. (See [27] for discussion of double-logarithmic corrections to DIS in the region $x \rightarrow 1$ and to timelike processes, and the need for resummations in these cases.) TMD factorization is required to treat the physics of the scaling violation in the high-energy regime, where transverse momentum ordering in the partonic initial state does not apply.

This is the motivation for the work we are going to describe in the next section, in which a quantitative step is taken toward going beyond fixed-order phenomenology, and using the high-precision combined measurements [18, 19] for determination of the nonperturbative TMD gluon density function.

\footnotetext{
${ }^{1}$ The three-loop coefficient agrees with the complete next-to-next-toleading-order (NNLO) calculation [26].
}

It is worth noting that similar motivation is at the basis of approaches such as those in [23]. These works go beyond fixed-order analyses by including perturbative resummations. However, they do not attempt to extract information on the TMD gluon density from experimental data.

\section{TMDs from the combined HERA data}

The combined measurements of proton's DIS structure functions at the HERA collider [18] provide highprecision data capable of constraining parton density functions over a broad range of the kinematic variables. These data have been widely used for determinations of the integrated (collinear) pdfs and for applications of these pdfs to LHC processes [14, 28]. Ref. [15] takes a first step to extend this approach by studying what can be learnt from the precision DIS data about hadron structure beyond collinear level, using the TMD factorization of Sec. 2 .

Phenomenological applications of this approach at HERA collider energies require matching of $x \ll 1$ contributions with finite- $x$ contributions. To this end, in [15] the evolution of the gluon density is obtained by combining the resummation of small- $x$ logarithmic contributions [29] with medium- $x$ and large- $x$ contributions to parton splitting [30] according to the CCFM evolution equations [31]. This is done via the exclusive parton-branching Monte Carlo implementation [32] of CCFM evolution.

The TMD gluon distribution at the initial scale of the evolution is determined from fits to precision DIS data. Fits are performed to the combined HERA charm-quark leptoproduction data [19] over the whole kinematic range of the measurement, corresponding to $Q^{2}>2.5 \mathrm{GeV}^{2}$, and to the $F_{2}$ structure function data [18] in the range $Q^{2}>\bar{Q}^{2}$, $x<\bar{x}$, where we take $\bar{Q}^{2}=5 \mathrm{GeV}^{2}, \bar{x}=5 \cdot 10^{-3}$. $^{2}$

\footnotetext{
${ }^{2}$ The restriction on the kinematic range for the $F_{2}$ data is motivated by the fact that the approach relies on perturbative factorization theorems, which classify higher-order corrections according to the logarithmic hierarchy based on high $Q^{2}$ and low $x$. The choice of the cuts $\bar{Q}, \bar{x}$ presented here may however be regarded as conservative. As discussed in [15], extensions to lower $Q^{2}$ and higher $x$ are possible - this may be part of programs for investigating future uses of HERA precision measurements [33]. See e.g. [34] for some of the issues involved in approaches to low $Q^{2}$ using unintegrated parton correlation functions.
} 

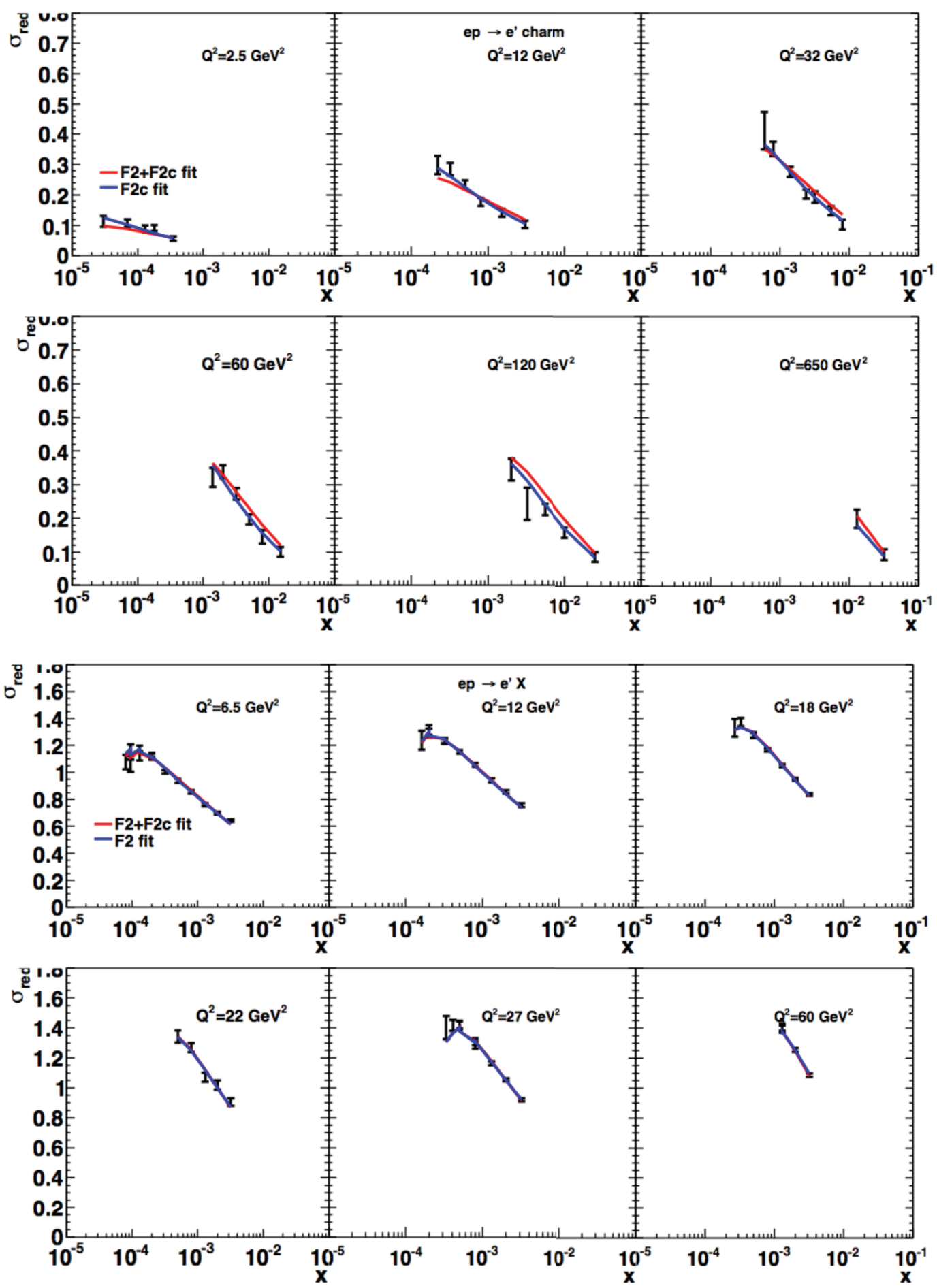

Figure 2. The fit [15] to DIS precision measurements: (top) charm leptoproduction data [19]; (bottom) inclusive structure function data [18].

The fits to the HERA measurements are obtained with the herafitter package $[14,17,18,35]$, treating the correlated systematic uncertainties separately from the uncorrelated statistical and systematic uncertainties.

The Monte Carlo implementation [32] of CCFM evolution includes two-loop running coupling, finite- $x$ gluon splitting and energy-momentum consistency constraint $[15,36]$. In addition to the gluon-induced process $\gamma^{*} g^{*} \rightarrow q \bar{q}$ the contribution from valence quarks is included via $\gamma^{*} q \rightarrow q$ by using a CCFM evolution of valence quarks [37]. ${ }^{3}$ Two different functional forms (with three and five parameters) for the starting gluon distribution are used. The corresponding results for the values of $\chi^{2}$ per degree of freedom are shown in Table 1 . This re-

\footnotetext{
${ }^{3}$ TMD sea quarks [38] are not yet included.
} 
Table 1. The values of $\chi^{2} / n d f$ [15] corresponding to the best fit for charm structure function $F_{2}^{(\mathrm{charm})}$, for inclusive structure function $F_{2}$, and for the combination of $F_{2}^{(\mathrm{charm})}$ and $F_{2}$.

\begin{tabular}{|c|c|c|c|}
\hline & $\chi^{2} / n d f\left(F_{2}^{\text {(charm) }}\right)$ & $\chi^{2} / n d f\left(F_{2}\right)$ & $\chi^{2} / n d f\left(F_{2}\right.$ and $\left.F_{2}^{\text {(charm) }}\right)$ \\
\hline 3-parameter & 0.63 & 1.18 & 1.43 \\
\hline 5-parameter & 0.65 & 1.16 & 1.41 \\
\hline
\end{tabular}
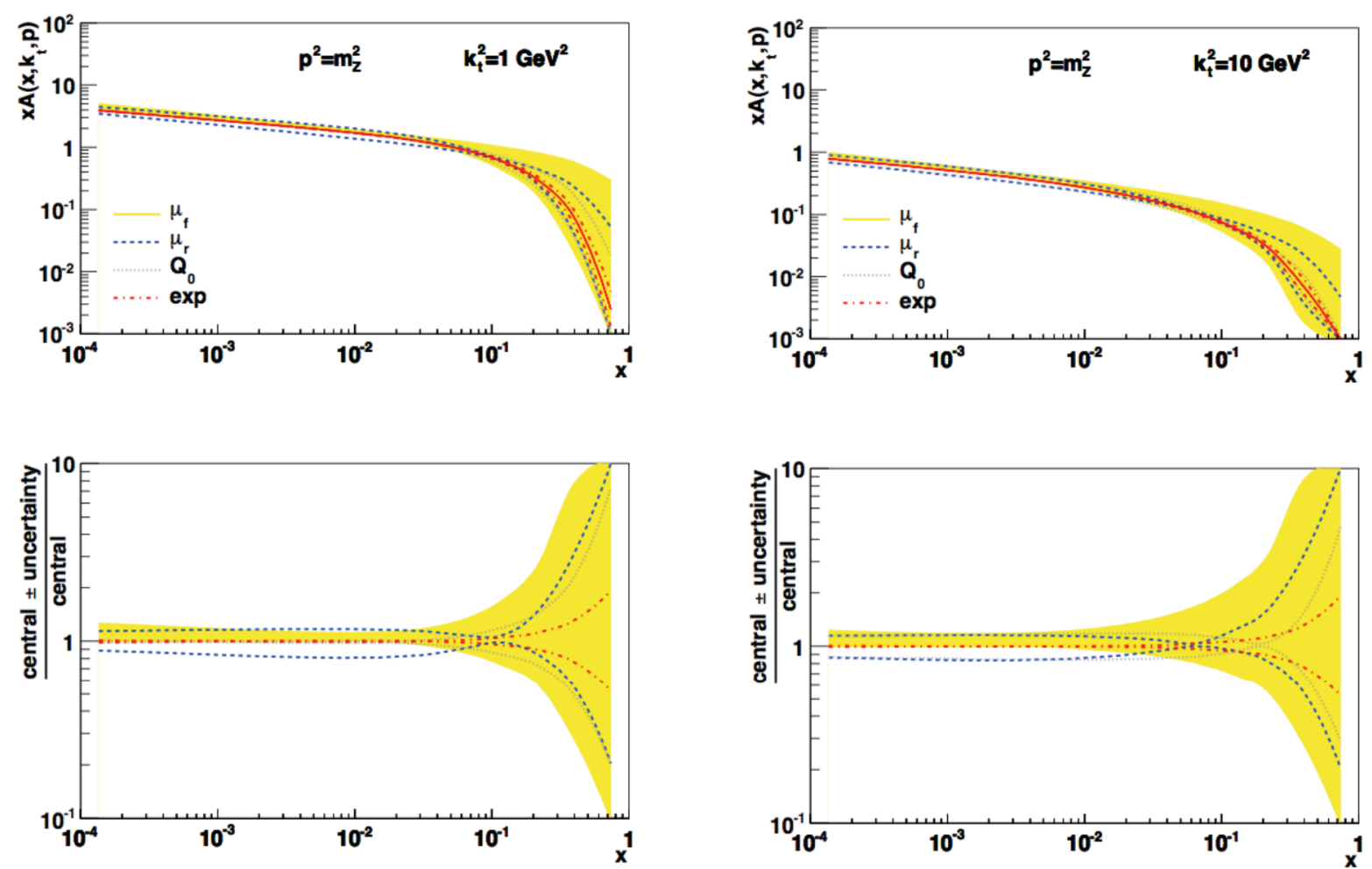

Figure 3. Experimental and theoretical uncertainties of the unintegrated TMD gluon density versus x for different values of transverse momentum at $p^{2}=m_{Z}^{2}$ [15]. The yellow band gives the uncertainty from the factorization scale variation, while the curves indicate the uncertainties from renormalization scale, starting scale, and experimental errors, as described in [15].

ports the $\chi^{2}$ per degree of freedom for the best fit to the charm structure function $F_{2}^{\text {(charm) }}$ [19], the inclusive structure function $F_{2}$ [18], and a combination of both. Fig. 2 shows the description of the charm leptoproduction and inclusive structure function measurements, by the individual fits and a combined fit. Plotted are the reduced cross sections defined in $[18,19]$.

We see from Table 1 that the best-fit $\chi^{2} / n d f$ is below 1 for the charm structure function, while it is around 1.18 for the inclusive structure function. This is in accord with expectations based on charm production coupling predominantly to gluons while the inclusive structure function couples to quark channels, for which sea quark distributions are not yet included at TMD level. Despite the restricted kinematic range of the experimental data analyzed, the great precision of the measurements provides a highly nontrivial test of the approach. Based on the fit to charm-quark and inclusive data, Ref. [15] presents two sets of TMD pdfs, JH-2013. It also presents experimental and theoretical uncertainties associated with these. An example is shown in Fig. 3, where different contributions to the uncertainty (both from experiment and from theory) are plotted for different values of transverse momentum at evolution scale equal to the $Z$ boson mass. The uncertainties are small for low $x$ values, while they become sizeable for high $x$ values, where there is little constraint from experimental data, and the theoretical accuracy of the calculation decreases.

\section{Discussion}

The work described in Sec. 3 is the first determination of the TMD gluon distribution which includes the precision DIS measurements and which provides experimental and theoretical uncertainties. As discussed earlier, despite the limited kinematic range of the $F_{2}$ data, $Q^{2}>5 \mathrm{GeV}^{2}$, $x<5 \cdot 10^{-3}$, their high precision implies a stringent test of the approach based on TMD factorization at high energy.

The method [15] for determining uncertainties associated with the TMD pdfs, implemented within herafitter [14], includes theoretical uncertainties on the TMD gluon density from variation of the factorization 

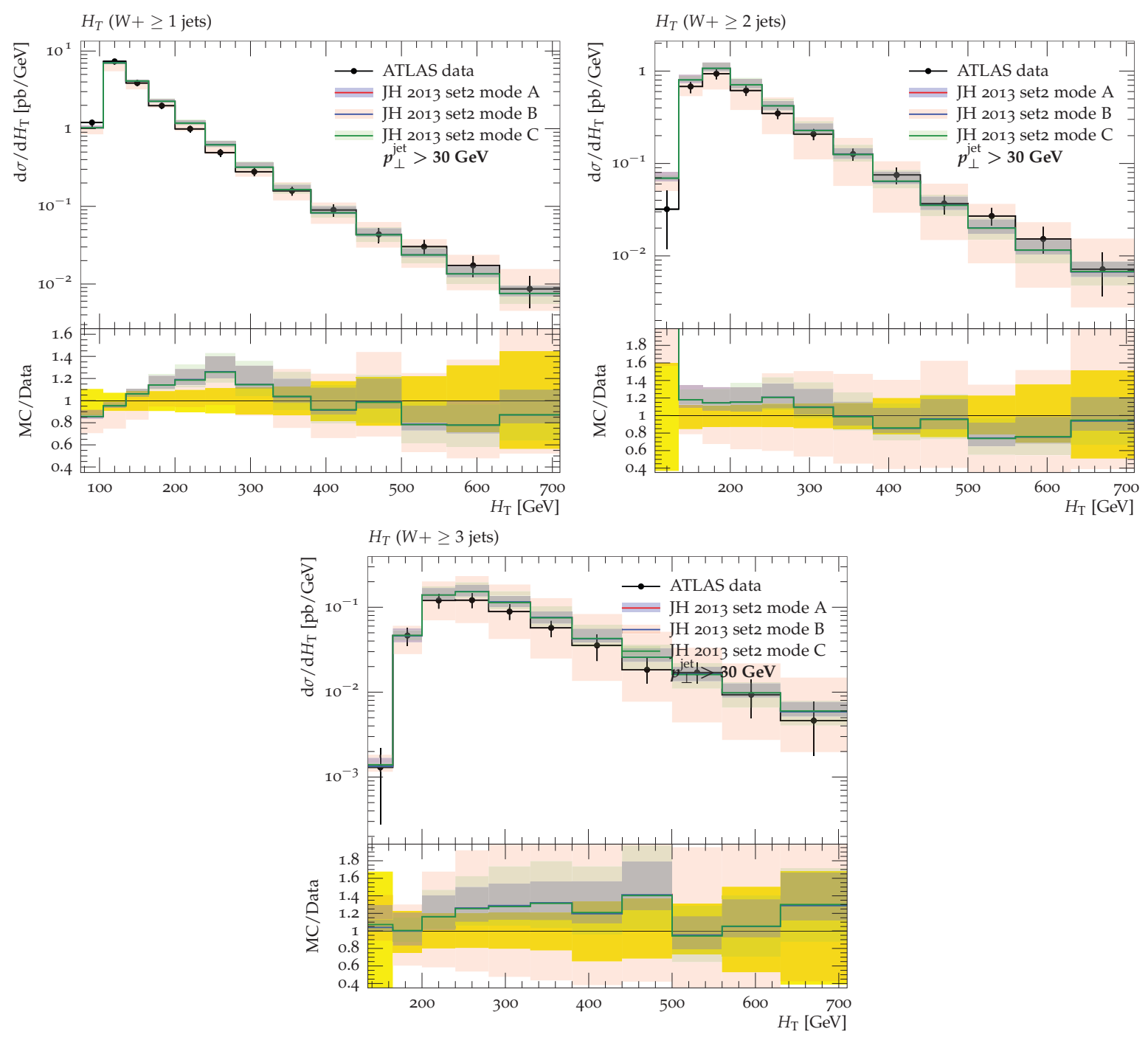

Figure 4. Total transverse energy $H_{T}$ distribution in final states with $W$-boson $+n$ jets at the $L H C$, for $n \geq 1, n \geq 2, n \geq 3$. The purple, pink and green bands correspond to the mode A, mode B and mode C, described in [42], to estimate theoretical uncertainties. The experimental data are from [43], with the experimental uncertainty represented by the yellow band.

scale and renormalization scale. This differs from that usually followed in determinations of ordinary, collinear pdfs from fixed-order perturbative treatments. In this case, no uncertainty on the pdfs is considered from scale variation. It is only when computing predictions for any specific observable that the theoretical uncertainty on the predictions is estimated by scale variation. In the approach [15] one is interested to study the uncertainty from varying scales in the theoretical calculation used to determine the pdf. For example in Fig. 3 the renormalization scale (blue dashed curves) and the factorization scale (yellow band) are varied by a factor of 2 above and below the central value. It can be interesting to extend this approach to collinear fits.

Having examined physics in the HERA region, one can ask whether this theoretical framework can be used to treat higher $p_{T}$ processes. To this end observe that, unlike forms of TMD factorization specifically designed for the low- $p_{T}$ region [7, 39, 40], the factorization [21] employed in the previous section is based on the high-energy expansion $\sqrt{s} \rightarrow \infty$ and it is valid for arbitrarily large momentum transfers. It is designed to take into account TMD physical effects which persist at high $p_{T}$ and can affect final states with high jet multiplicities [41]. We further note that the implementation [32] of TMD evolution as an exclusive branching Monte Carlo process explicitly provides, along with the parton density, the detailed structure of the final states. ${ }^{4}$ Based on these observations, Ref. [42] investigates the applicability of the TMD theoretical framework to large- $p_{T}$ processes, by studying multi-jet final states associated with Drell-Yan (DY) production of electroweak gauge bosons at the LHC.

Fig. 4 shows predictions based on the TMD distribution functions discussed in the previous section for the total transverse energy $H_{T}$ distribution in final states with $W$ boson $+n$ jets, with $n=1,2,3$, at the LHC. For compar-

\footnotetext{
${ }^{4}$ This is a specific feature of the approach [32] and distinguishes it both from ordinary parton-shower Monte Carlo generators and from TMD resummation programs such as $[10,11]$.
} 

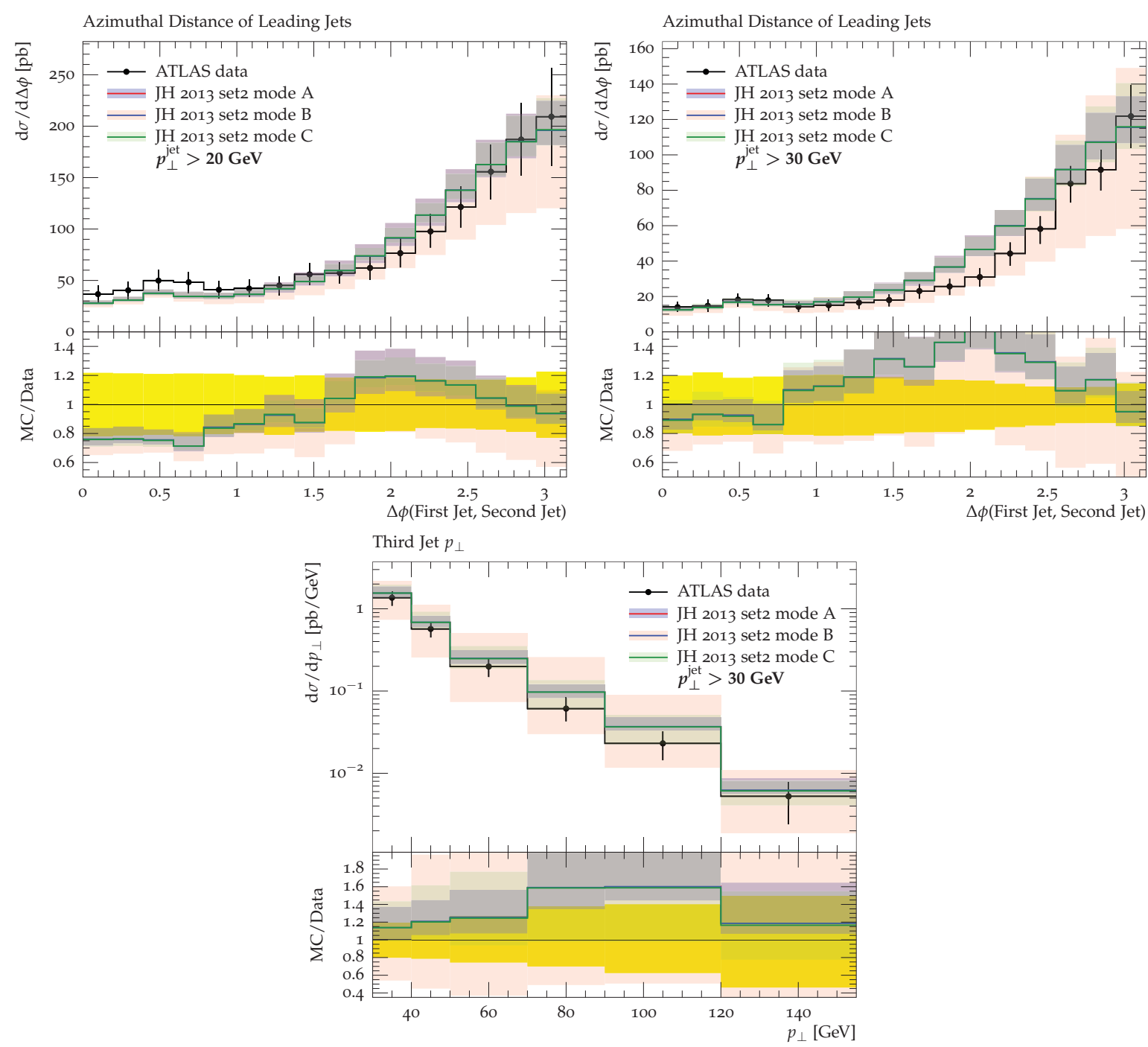

Figure 5. (top) Azimuthal distance of the two leading jets associated with $\mathrm{W}$-bosons, for $p_{T}>20 \mathrm{GeV}$ (left) and $p_{T}>30 \mathrm{GeV}$ (right); (bottom) transverse momentum of the third jet $\left(p_{T}>30 \mathrm{GeV}\right)$. The purple, pink and green bands correspond to the mode A, mode $B$ and mode $C$, described in [42], to estimate theoretical uncertainties. The experimental data are from [43], with the experimental uncertainty represented by the yellow band.

ison we plot the experimental measurements [43] (jet rapidity $|\eta|<4.4$, jet transverse momentum $\left.p_{T}>30 \mathrm{GeV}\right)$. The main features of the $H_{T}$ distribution are described by the predictions, including the case of higher jet multiplicities. The theoretical uncertainties are shown according to three different modes as discussed in [42]: mode A (purple band in the plots) includes uncertainties due to the renormalization scale, starting evolution scale, and experimental errors, while mode B (pink band in the plots) and mode $\mathrm{C}$ (green band in the plots) also include factorization scale uncertainties. ${ }^{5}$ The theoretical uncertainties are larger for larger $H_{T}$ (increasing $x$ ) and, at fixed $H_{T}$, for higher jet multiplicities. Ref. [42] also examines the transverse momentum spectra of the individual jets, and the angular correlations between the jets and of the jets with the

\footnotetext{
${ }^{5}$ Mode $\mathrm{B}$ is to be regarded as the most conservative estimate and gives the largest uncertainty bands. Mode $\mathrm{C}$ is most closely related to to the estimation of uncertainties in standard, collinear calculations, and gives bands intermediate between the other two modes. See [42] for details.
}

vector boson. Within the uncertainties, a good description of the measurements $[43,44]$ is found. Fig. 5 shows the azimuthal distance between the two leading jets, and the transverse momentum of the third jet.

The main advantage of the exclusive TMD approach to $W$-boson $+n$ jets proposed in [42] is the possibility to construct a formalism which interpolates from low $p_{T}$ to high $p_{T}$, and incorporates all-order coherence effects $[31,41,45]$ associated with multiple soft-gluon emission at finite angle, possibly enhanced in events with large rapidity intervals between final-state particles [46]. These effects are beyond treatments based on next-to-leadingorder perturbation theory matched with collinear parton showers [47]. With automated methods for off-shell multileg calculations currently being developed [48], the TMD approach could become a general tool for phenomenological analyses of complex hadroproduction final states at the LHC. 
A general motivation for TMD approaches comes from the observation of sizeable kinematic corrections to showering algorithms due to collinearity approximations [49]. This, for instance, underlines the relevance of Monte Carlo generators $[50,51]$ that aim to go beyond these approximations. In the specific case of vector boson + jets, TMD corrections to showering [52] may affect the interpretation of $W+2 j$ measurements [53] as a signal for double parton scattering.

It should be noted that, unlike the HERA measurements in Sec. 3, the longitudinal momentum fractions $x$ sampled in the $W$-boson + jets cross sections at the LHC are not very small. Moreover, quark density contributions matter at TMD level. For these reasons, $W+$ jets pushes the limits of the approach, in a manner which can be controlled using the estimation of theoretical and experimental uncertainties on TMD distributions. The results are however encouraging, and sufficiently general to be of interest to any approach that employs TMD formalisms in QCD to go beyond fixed-order perturbation theory and appropriately take account of nonperturbative effects. This can be relevant both to precision studies of Standard Model physics and to new physics searches for which vector boson plus jets production is an important background. In particular, future applications may employ vector boson $p p$ data to advance our knowledge of transverse momentum parton distributions.

Acknowledgments. We are grateful to F. Fabbri, P. Giacomelli, V. Radescu, G. Watt and the organizing team for the very kind invitation to an excellent symposium.

\section{References}

[1] S. Mert Aybat and T.C. Rogers, Phys. Rev. D83 (2011) 114042.

[2] F. Hautmann, H. Jung, M. Krämer, P.J. Mulders, E.R. Nocera, T.C. Rogers and A. Signori, arXiv: 1408.3015 [hep-ph].

[3] M. Anselmino, M. Boglione, J. Gonzalez H., S. Melis and A. Prokudin, JHEP 1404 (2014) 005.

[4] A. Signori, A. Bacchetta, M. Radici and G. Schnell, JHEP 1311 (2013) 194.

[5] HERMES Coll. (A. Airapetian et al.), Phys. Rev. D87 (2013) 074029.

[6] COMPASS Coll. (C. Adolph et al.), Eur. Phys. J. C 73 (2013) 2531.

[7] J.C. Collins, D.E. Soper and G. Sterman, Nucl. Phys. B250 (1985) 199.

[8] J.C. Collins, Foundations of perturbative QCD, CUP 2011.

[9] S. Mert Aybat, J.C. Collins, J.-W. Qiu and T.C. Rogers, Phys. Rev. D85 (2012) 034043.

[10] F. Landry, R. Brock, P.M. Nadolsky and C.P. Yuan, Phys. Rev. D67 (2003) 073016.

[11] M. Guzzi, P.M. Nadolsky and B. Wang, Phys. Rev. D90 (2014) 014030.
[12] D0 Coll. (V.M. Abazov et al.), Phys. Rev. Lett. 106 (2011) 122001.

[13] ATLAS Coll. (G. Aad et al.), Phys. Lett. B705 (2011) 415.

[14] "HERAFitter" Open Source QCD Fit Project, arXiv: 1410.4412 [hep-ph]; https: //www.herafitter.org/ (2014).

[15] F. Hautmann and H. Jung, Nucl. Phys. B883 (2014) 1.

[16] F. Hautmann and H. Jung, arXiv: 1206.1796 [hep-ph].

[17] H1 Coll. (F. Aaron et al.), Eur. Phys. J. C64 (2009) 561.

[18] H1 and ZEUS Coll. (F. Aaron et al.), JHEP 1001 (2010) 109.

[19] H1 and ZEUS Coll. (H. Abramowicz et al.), Eur. Phys. J. C 73 (2013) 2311.

[20] S. Catani and F. Hautmann, Nucl. Phys. B427 (1994) 475; Phys. Lett. B315 (1993) 157.

[21] S. Catani, M. Ciafaloni and F. Hautmann, Phys. Lett. B307 (1993) 147; Nucl. Phys. B366 (1991) 135; Phys. Lett. B242 (1990) 97.

[22] V.S. Fadin and L.N. Lipatov, Phys. Lett. B429 (1998) 127; G. Camici and M. Ciafaloni, Phys. Lett. B430 (1998) 349.

[23] G. Altarelli, R. Ball and S. Forte, Nucl. Phys. B799 (2008) 199; M. Ciafaloni, D. Colferai, G.P. Salam and A. Stasto, JHEP 0708 (2007) 046; R.S. Thorne, Phys. Rev. D60 (1999) 054031; R.K. Ellis, F. Hautmann and B.R. Webber, Phys. Lett. B 348 (1995) 582.

[24] G. Curci, W. Furmanski and R. Petronzio, Nucl. Phys. B175 (1980) 27.

[25] E. Zijlstra and W.L. van Neerven, Nucl. Phys. B383 (1992) 525.

[26] S. Moch, J. Vermaseren and A. Vogt, Nucl. Phys. B724 (2005) 3.

[27] A. Vogt et al., arXiv: 1212.2932 [hep-ph].

[28] S. Alekhin et al., arXiv:1101.0536 [hep-ph].

[29] L.N. Lipatov, Phys. Rept. 286 (1997) 131; V. S. Fadin, E. A. Kuraev and L. N. Lipatov, Phys. Lett. B 60 (1975) 50; I. I. Balitsky and L. N. Lipatov, Sov. J. Nucl. Phys. 28 (1978) 822.

[30] V.N. Gribov and L.N. Lipatov, Sov. J. Nucl. Phys. 15 (1972) 438; G. Altarelli and G. Parisi, Nucl. Phys. B126 (1977) 298; Yu.L. Dokshitzer, Sov. J. Nucl. Phys. 46 (1977) 641.

[31] M. Ciafaloni, Nucl. Phys. B296 (1988) 49, S. Catani, F. Fiorani and G. Marchesini, Nucl. Phys. B336 (1990) 18, G. Marchesini, Nucl. Phys. B445 (1995) 49.

[32] F. Hautmann, H. Jung and S. Taheri Monfared, Eur. Phys. J. C 74 (2014) 3082.

[33] Workshop "Future Physics with HERA Data for Current and Planned Experiments", DESY, November 2014.

[34] F. Hautmann and D.E. Soper, Phys. Rev. D75 (2007) 074020; Phys. Rev. D63 (2000) 011501; Phys. Rev. Lett. 81 (1998) 3333; F. Hautmann, Phys. Lett. B643 
(2006) 171; arXiv:0812.2873 [hep-ph].

[35] F. James and M. Roos, Comput. Phys. Commun. 10 (1975) 343.

[36] F. Hautmann and H. Jung, PoS DIS2013 (2013) 053; PoS DIS2014 (2014) 042.

[37] M. Deak, F. Hautmann, H. Jung and K. Kutak, arXiv: 1012.6037 [hep-ph]; Eur. Phys. J. C 72 (2012) 1982.

[38] F. Hautmann, M. Hentschinski and H. Jung, Nucl. Phys. B865 (2012) 54; arXiv: 1205.6358 [hep-ph]; arXiv:1207.6420 [hep-ph]; arXiv: 1209.6305 [hep-ph].

[39] M.G. Echevarria, A. Idilbi and I. Scimemi, JHEP 01207 (2012) 002; Phys. Lett. B 726 (2013) 795; Phys. Rev. D90 (2014) 014003; arXiv: 1311.1547 [hep-ph]; arXiv: 1310.8541 [hep-ph].

[40] M.G.A. Buffing, A. Mukherjee and P.J. Mulders, arXiv: 1409.7257 [hep-ph]; Phys. Rev. D88 (2013) 054027; Phys. Rev. D86 (2012) 074030; M.G.A. Buffing and P.J. Mulders, Phys. Rev. Lett. 112 (2014) 092002; P.J. Mulders, Pramana 72 (2009) 83.

[41] F. Hautmann, Acta Phys. Polon. B 40 (2009) 2139; arXiv:0909.1240 [hep-ph]; F. Hautmann and H. Jung, JHEP 0810 (2008) 113; arXiv: 0804. 1746 [hep-ph]; arXiv:0712.0568 [hep-ph].
[42] S. Dooling, F. Hautmann and H. Jung, Phys. Lett. B 736 (2014) 293.

[43] ATLAS Coll. (G. Aad et al.), Phys. Rev. D 85 (2012) 092002.

[44] CMS Coll. (V. Khachatryan et al.), arXiv: 1406.7533 [hep-ex].

[45] G. Marchesini and B.R. Webber, Nucl. Phys. B386 (1992) 215, Nucl. Phys. B349 (1991) 617.

[46] F. Hautmann, Acta Phys. Polon. B 44 (2013) 761; arXiv: 1304.8133 [hep-ph]; arXiv:0909.1250 [hep-ph].

[47] S. Höche, F. Krauss, M. Schönherr and F. Siegert, Phys. Rev. Lett. 110 (2013) 052001.

[48] A. van Hameren, P. Kotko and K. Kutak, JHEP 1301 (2013) 078; A. van Hameren, K. Kutak and T. Salwa, Phys. Lett. B 727 (2013) 226.

[49] S. Dooling et al., Phys. Rev. D87 (2013) 094009; arXiv: 1304.7180 [hep-ph]; PoS DIS2013 (2013) 156; F. Hautmann and H. Jung, Eur. Phys. J. C 72 (2012) 2254; Nucl. Phys. Proc. Suppl. 234 (2013) 51.

[50] H. Jung et al., Eur. Phys. J. C 70 (2010) 1237.

[51] S. Jadach and M. Skrzypek, Acta Phys. Polon. B 40 (2009) 2071.

[52] F. Hautmann, arXiv: 1205.5411 [hep-ph].

[53] ATLAS Coll. (G. Aad et al.), New. J. Phys. 15 (2013) 033038, arXiv: 1301.6872 [hep-ex]. 